\title{
Simultaneous Determination of Amphenicols and Metabolites in Animal-Derived Foods Using Ultrahigh-Performance Liquid Chromatography-Tandem Mass Spectrometry
}

\author{
Xinyi Wu, Xixi Shen, Xiangyue Cao, Rongrong Nie, Haonan Zhang, Changbo Tang, \\ and Wei Wang $(10$
}

Key Laboratory of Meat Processing and Quality Control, MOE, Key Laboratory of Meat Processing, MARA, Jiangsu Collaborative Innovation Center of Meat Production and Processing, Quality and Safety Control, College of Food Science and Technology, Nanjing Agricultural University, Nanjing 210095, China

Correspondence should be addressed to Wei Wang; wangwei821220@njau.edu.cn

Received 9 August 2021; Revised 15 September 2021; Accepted 22 October 2021; Published 3 November 2021

Academic Editor: Barbara Bojko

Copyright (c) 2021 Xinyi Wu et al. This is an open access article distributed under the Creative Commons Attribution License, which permits unrestricted use, distribution, and reproduction in any medium, provided the original work is properly cited.

Amphenicols are widely used to prevent and treat animal diseases. However, amphenicol residues accumulate in livestock and poultry and harm consumers. We hypothesized that one can combine solid-phase extraction (SPE) with ultrahigh-performance liquid chromatography-tandem mass spectrometry (UHPLC-MS/MS) to simultaneously determine amphenicols and metabolites in pork, beef, lamb, chicken, and their products and meet government regulations for maximum residue limits. We extracted crude samples with ethyl acetate and ammonia water (98:2, v/v), purified the samples with a CNW Si SPE column, defatted the samples with acetonitrile-saturated $n$-hexane, and then determined the resulting analytes by UHPLC-MS/MS. The limit of detection of the analytes in livestock and poultry meat was $0.03-1.50 \mu \mathrm{g} / \mathrm{kg}$, and the limit of quantification was $0.05-5.00 \mu \mathrm{g} / \mathrm{kg}$. Measured chloramphenicol, thiamphenicol, and florfenicol concentrations were linear over the range $0.50-50 \mu \mathrm{g} / \mathrm{kg}$; and the florfenicol amine concentration was linear over the range $5.00-200 \mu \mathrm{g} / \mathrm{kg}$ (all with correlation coefficients $>0.9990$ ). The recovery of the spiked samples was between $72 \%$ and $120 \%$. The intraday relative standard deviation (RSD) ranged from $1 \%$ to $9 \%$, and the interday RSD ranged from $1 \%$ to $12 \%$. Based on the above results, the current method is sensitive, accurate, and reproducible with the detection limits being well below the maximum residue limits as per Chinese standard GB 31650-2019, and thus, our research hypothesis could be confirmed.

\section{Introduction}

China produces and consumes a large quantity of livestock and poultry meat; corresponding quality and safety problems harm consumer health and create barriers to the import and export trade [1]. Among these quality and safety problems, veterinary drug residues are especially pertinent. Chloramphenicol (CAP), thiamphenicol (TAP), and florfenicol (FF) are amphenicols, substances that are widely used in livestock and poultry breeding and production. These substances have broad-spectrum antibacterial properties, are low cost, and have high potency [2-4]. However, CAP is highly toxic to the human hematopoietic system and can cause side effects such as aplastic anemia $[5,6]$. As a derivative of CAP, TAP replaces the para-nitro group of CAP with a methanesulfonyl group and is less toxic than CAP but can inhibit the production of red blood cells, white blood cells, and platelets $[7,8]$. FF is synthesised from TAP by replacing the 3-hydroxy group with fluorine; it is an animalspecific amphenicol and has higher antibacterial activity than CAP and TAP but has embryo toxicity $[9,10]$. FF is metabolized to florfenicol amine (FFA) via bioconversion pathways in treated animals (Figure 1) [11]. Therefore, to regulate the use of toxic amphenicols and reduce their prevalence in livestock and poultry products, the use of amphenicols is strictly restricted by laws and regulations. 


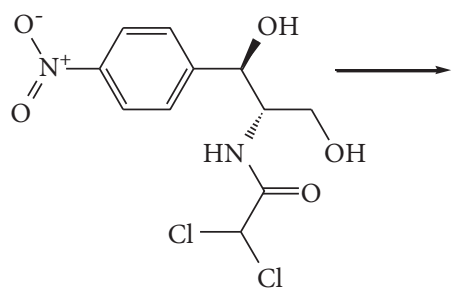

Chloramphenicol (CAP)<smiles>CS(=O)(=O)c1ccc([C@@H](O)[C@H](CO)NC(=O)C(Cl)Cl)cc1</smiles>

Thiamphenicol (TAP)<smiles>CS(=O)(=O)c1ccc([C@@H](O)[C@H](CF)NC(=O)C(Cl)Cl)cc1</smiles>

Florfenicol
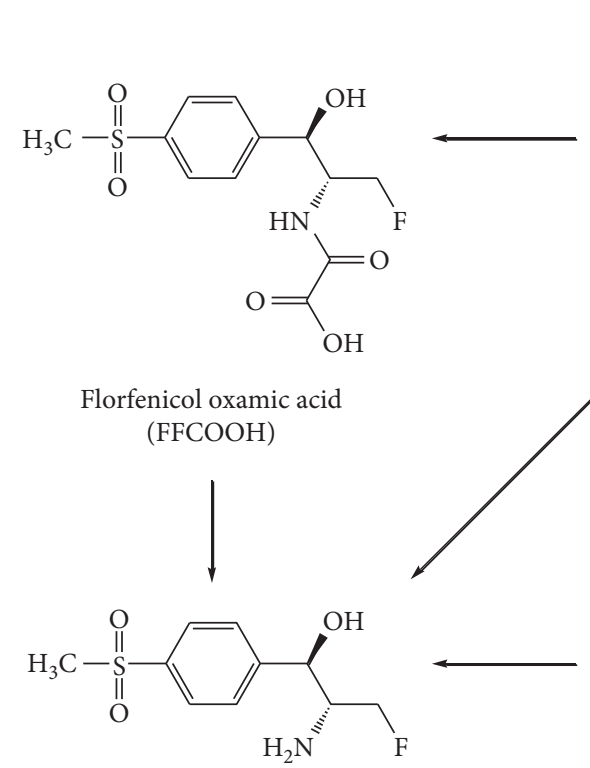

Florfenicol amine (FFA)

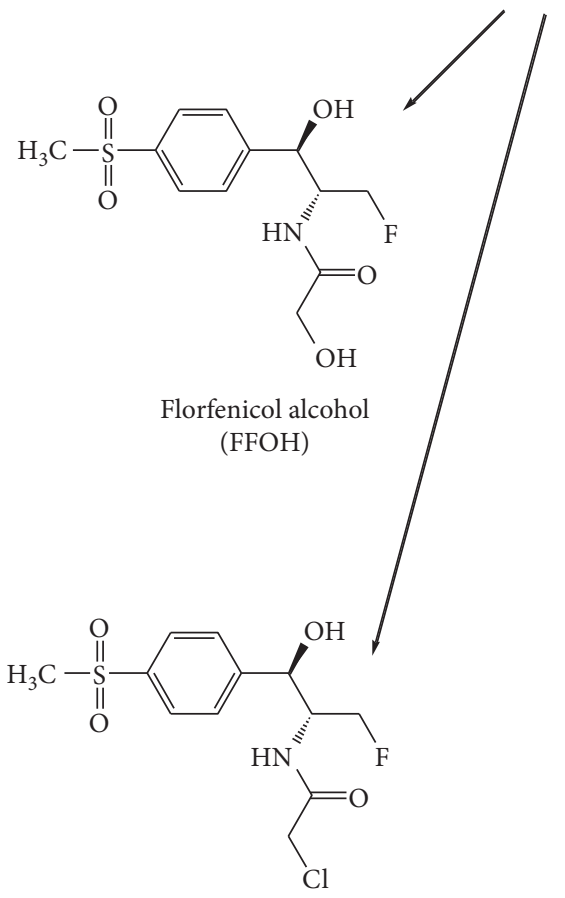

Monochloroflorfenicol

(FFCl)

FIgURE 1: Chemical structures of amphenicols and metabolic route of FF.

Currently, China, the United States, the European Union, Japan, and other countries have banned the use of CAP in animal feeds. As shown in Table 1, regulations in China, the United States (US), and the European Union (EU) have stipulated that the maximum residue limits (MRLs) of TAP in animal-derived foods such as the muscle, fat, liver, kidney, and milk are $50 \mu \mathrm{g} / \mathrm{kg}$; and the MRLs of FF (calculated as the sum of both FF and FFA) in the muscle, liver, kidney, skin, and fat are $100-3700 \mu \mathrm{g} / \mathrm{kg}$ [12-17]. TAP is not recommended for animals from which eggs are produced for human consumption, and FF is not recommended for animals from which milk and eggs are produced for human consumption in China and the EU

Various approaches for the determination of amphenicols in animal-derived foods are available in the literature and apply detection systems such as biosensors [18-21], enzyme-linked immunosorbent assays [22, 23], high-performance liquid chromatography (HPLC) [24-26], liquid chromatography-tandem mass spectrometry (LC-MS/MS) [27-30], gas chromatography (GC) [31], and GC-MS/MS $[32,33]$. Among them, LC-MS/MS is the main detection and positive confirmation method for amphenicols. Ultrahighperformance liquid chromatography (UHPLC)-MS/MS is an LC-MS/MS technology that is more suitable than conventional LC-MS/MS for the analysis and detection of amphenicols because of a short analysis time, higher separation efficiency, and higher sensitivity. Currently, several analytical methods have been reported for the analysis of some of amphenicols and metabolites [28, 30, 34, 35]. In addition, these methods are usually developed for one or two matrices [7, 36, 37]. To our knowledge, there are few reports on simultaneously detecting the entire family of amphenicols and metabolites in animal-derived foods. In this study, we combined solid-phase extraction (SPE) with UHPLCMS/MS in both negative and positive electrospray ionization (ESI) mode for simultaneous determination of CAP, TAP, FF, and FFA in pork, beef, lamb, chicken, and their products under the optimal experimental conditions. Our goal is to promote the development of international trade in animalderived foods, provide references for quality and safety monitoring, and standardize supervision and testing methods of animal-derived foods. 
TABLE 1: Comparison of the MRLs for amphenicols in China, the US, and the EU.

\begin{tabular}{|c|c|c|c|c|c|}
\hline \multirow{2}{*}{ Analyte } & \multirow{2}{*}{ Species } & \multirow{2}{*}{ Target tissue } & \multicolumn{3}{|c|}{ MRL $(\mu \mathrm{g} / \mathrm{kg})$} \\
\hline & & & China & US & EU \\
\hline TAP & All species & All tissues & 50 & & 50 \\
\hline \multirow{14}{*}{ FF (sum of FF and FFA) } & \multirow{4}{*}{ Porcine } & Muscle & 300 & 200 & 300 \\
\hline & & Skin and fat & 500 & & 500 \\
\hline & & Liver & 2000 & 2500 & 2000 \\
\hline & & Kidney & 500 & & 500 \\
\hline & \multirow{3}{*}{ Bovine } & Muscle & 200 & 300 & 200 \\
\hline & & Liver & 3000 & 3700 & 3000 \\
\hline & & Kidney & 300 & & 300 \\
\hline & \multirow{3}{*}{ Ovine caprine } & Muscle & 200 & & 200 \\
\hline & & Liver & 3000 & & 3000 \\
\hline & & Kidney & 300 & & 300 \\
\hline & \multirow{4}{*}{ Poultry } & Muscle & 100 & & 100 \\
\hline & & Skin and fat & 200 & & 200 \\
\hline & & Liver & 2500 & & 2500 \\
\hline & & Kidney & 750 & & 750 \\
\hline
\end{tabular}

\section{Materials and Methods}

2.1. Chemicals and Reagents. CAP, TAP, FF, FFA, and CAPD5 standards were obtained from Dr. Ehrenstorfer $\mathrm{GmbH}$ (Augsburg, Germany). FFA-D3 standard was obtained from Toronto Research Chemicals (Toronto, ON, Canada). HPLC-grade acetonitrile (ACN), methanol, ethyl acetate, and $n$-hexane were supplied by Merck (Darmstadt, Germany). Reagent grade ammonium hydroxide, HPLC-grade acetone, and formic acid were supplied by Sinopharm Chemical Reagent Co. (Shanghai, China). Water was purified with an Arium Pro ultrapure water purification system (Sartorius, Göttingen, Germany).

2.2. Preparation of Standard Stock and Working Solutions. Standard stock solutions of CAP, TAP, FF, FFA, CAP-D5, and FFA-D3 were prepared at a concentration of $1000 \mu \mathrm{g} /$ $\mathrm{mL}$ by dissolving each analyte in methanol. All the aforementioned standard stock solutions were stored at $-20^{\circ} \mathrm{C}$ for up to 6 mo.

Mixed standard working solutions of CAP, TAP, FF, and FFA at a concentration of $10 \mu \mathrm{g} / \mathrm{mL}$ were prepared by diluting the standard stock solutions with methanol. An internal standard working solution at a concentration of $1.0 \mu \mathrm{g} / \mathrm{mL}$ was prepared by diluting the internal standard stock solutions with methanol. The aforementioned standard working solutions were stored at $-20^{\circ} \mathrm{C}$ for up to 3 mo.

2.3. UHPLC-MS/MS Instrumentation and Operating Conditions. A Thermo Scientific Vanquish ultrahigh-performance liquid chromatograph coupled to a Thermo Scientific TSQ Quantis mass spectrometer (Thermo Fisher Scientific, Waltham, MA, USA) was used. Separation was performed with a Waters Acquity UPLC high-strength silica (HSS) C18 column $(50 \mathrm{~mm} \times 2.1 \mathrm{~mm}, 1.8 \mu \mathrm{m}$; Waters, Milford, MA, USA), with the front end connected to a Waters Acquity UPLC HSS C18 VanGuard precolumn ( $5 \mathrm{~mm} \times 2.1 \mathrm{~mm}, 1.8 \mu \mathrm{m}$; Waters, Milford, MA, USA). The column temperature was $40^{\circ} \mathrm{C}$, and the sample injection volume was $2.0 \mu \mathrm{L}$. Gradient elution was performed with water and ACN as the mobile phases, at a flow rate of $0.3 \mathrm{~mL} /$ min: $0-4 \mathrm{~min}, 4 \%(\mathrm{v} / \mathrm{v}) \mathrm{ACN}$ increased to $96 \%(\mathrm{v} / \mathrm{v})$; 4-6 min, 96\% (v/v) ACN; and 6-10 min, 96\% (v/v) ACN decreased to $4 \%(\mathrm{v} / \mathrm{v})$.

The MS/MS was equipped with an ESI source operating in the positive ionization (PI) mode, negative ionization (NI) mode, and selected reaction monitoring mode. CAP, TAP, FF, and CAP-D5 were analyzed in the NI mode, whereas FFA and FFA-D3 were analyzed in the PI mode. The ESI source was operated with the following capillary voltage: $3.5 \mathrm{kV}$ in PI mode and $2.5 \mathrm{kV}$ in NI mode; sheath gas: $50 \mathrm{Arb}$; auxiliary gas: $10 \mathrm{Arb}$; ion transfer tube temperature: $325^{\circ} \mathrm{C}$; and vaporizer temperature: $350^{\circ} \mathrm{C}$.

2.4. Sample Preparation. Blank samples used in the validation procedure were obtained from the Supervision, Inspection, and Testing Center for Quality of Meat Products (Nanjing, China). Pork, beef, lamb, and chicken muscle were chopped and homogenized by an HM6300 intelligent homogenizer (Lab Precision Beijing Technology Co., LTD, Beijing, China) at $10,000 \mathrm{r} / \mathrm{min}$, and aliquots of $5.0 \mathrm{~g}$ were weighed in a $50-\mathrm{mL}$ polypropylene centrifuge tube. Samples were spiked with $10 \mu \mathrm{L}$ of the internal standard working solution, corresponding to a concentration of $2 \mu \mathrm{g} / \mathrm{kg}$ of CAP-D5 and FFA-D3 in tissue. Then $15 \mathrm{~mL}$ of ethyl acetate with $2 \%(\mathrm{v} / \mathrm{v})$ ammonia were added. The mixture was thoroughly homogenized using an IKA Vortex 3 (IKA, Staufen, Germany). After mixing, the homogenate was centrifuged at $10,621 \mathrm{~g}$ for $5 \mathrm{~min}$ at $4^{\circ} \mathrm{C}$, and the supernatant was transferred into a $50-\mathrm{mL}$ polypropylene tube. The extraction step was repeated by the addition of $15 \mathrm{~mL}$ of ethyl acetate with $2 \%(\mathrm{v} / \mathrm{v})$ ammonia, shaken, and centrifuged again under the same conditions. The resulted supernatant was merged with the previous extract in the $50 \mathrm{~mL}$ polypropylene tube, and the extracts were evaporated to dryness at $40^{\circ} \mathrm{C}$ for $2.5 \mathrm{~h}$ under a nitrogen stream with an N-EVAP11 nitrogen evaporator (Organomation, Berlin, MA, USA). 
The residual solution was dissolved in acetone $/ n$-hexane (1:9, v/v; $5 \mathrm{~mL})$ and loaded onto a CNW Si SPE (200 mg) cartridge, which was preconditioned with water $(5 \mathrm{~mL})$ and acetone $/ n$-hexane $(1: 9, \mathrm{v} / \mathrm{v} ; 5 \mathrm{~mL})$. The cartridge was eluted with acetone $/ n$-hexane $(6: 4, \mathrm{v} / \mathrm{v} ; 5 \mathrm{~mL})$, and then the eluate was evaporated to dryness at $40^{\circ} \mathrm{C}$ for $10 \mathrm{~min}$ under a nitrogen stream. The residual solution was reconstituted by adding $1.0 \mathrm{~mL}$ of $\mathrm{ACN}$-water (50:50; v/v) and then vortexed for $1 \mathrm{~min}$. Next, $5 \mathrm{~mL}$ of hexane saturated with pure ACN was added to the tube, and the mixture was vortexed for $30 \mathrm{~s}$. After centrifugation for $1 \mathrm{~min}$ at $10,621 \mathrm{~g}$, the hexane layer was discarded. This defatting step was repeated twice. Finally, an aliquot of $1 \mathrm{~mL}$ of the bottom layer was poured through a $0.22 \mu \mathrm{m}$ filter into an autosampler vial, and then $2 \mu \mathrm{L}$ of the liquid in the aforementioned vial was analyzed using UHPLC-MS/MS.

\section{Method Validation}

3.1. Determination of Linearity, Limit of Detection, and Limit of Quantification of Amphenicols. A series concentration of matrix-matched standard solution was prepared by diluting the mixed standard working solutions with the blank matrix extract. Among them, CAP, TAP, and FF were set at 0.5, 1.0, $5.0,10.0$, and $50.0 \mu \mathrm{g} / \mathrm{kg}$ within the range $0.5-50.0 \mu \mathrm{g} / \mathrm{kg}$, and FFA was set at 5.0, 10.0, 50.0, 100.0, and $200.0 \mu \mathrm{g} / \mathrm{kg}$ within the range $5.0-200.0 \mu \mathrm{g} / \mathrm{kg}$ (the concentrations of CAP-D5 and FFA-D3 were $10 \mu \mathrm{g} / \mathrm{L}$ ). The standard curves were constructed using the mass concentration of amphenicols $(\mu \mathrm{g} / \mathrm{kg})$ as the abscissa $(x)$ and the analyte/internal standard peak area ratio versus as the ordinate $(y)$, and the squares of the correlation coefficients $\left(R^{2}\right)$ were calculated.

Negative livestock and poultry meat were added to the internal standard working solution and the mixed standard working solution and then detected with reference to the method described above. The limit of detection (LOD) was the minimum added concentration when the signalto-noise ratio $(S / N) \geq 3$, and the limit of quantification (LOQ) was the minimum added concentration when S/ $\mathrm{N} \geq 10$ [38].

3.2. Recovery and Precision Test. Solutions were spiked into livestock and poultry meat as follows: standard working solutions of CAP, TAP, and FF at three concentration levels $(1,5$, and $10 \mu \mathrm{g} / \mathrm{kg})$ and the standard working solution of FFA at three concentration levels $(5,10$, and $50 \mu \mathrm{g} / \mathrm{kg}$ ). Pretreatment was performed in accordance with the method described above, and each concentration was set in six parallels. The recovery was calculated as follows:

$$
\text { recovery }(\%)=\frac{C_{E}}{C_{M}} \times 100 \% \text {, }
$$

where $C_{E}$ is the experimental concentration determined from the calibration curve and $C_{M}$ is the spiked concentration. The precision was determined by calculating the relative standard deviation (RSD). Samples with three levels of spiked concentrations were measured in the same day and in six consecutive days, and then the intraday RSD and the interday RSD were calculated. The RSD was calculated as follows:

$$
\operatorname{RSD}(\%)=\frac{\mathrm{SD}}{C_{A}} \times 100 \%,
$$

where $\mathrm{SD}$ is the standard deviation and $C_{A}$ is the average of the experimental concentration determined from the calibration curve.

3.3. Application to Livestock Meat, Poultry Meat, and their Products. To validate our strategy, 28 real samples of commercial livestock meat, poultry meat, and their products from various locations in China were used to detect amphenicols. Amphenicols were extracted from the samples as previously described, and the extracts were analyzed with UHPLC-MS/MS.

3.4. Data Analysis. TraceFinder 4.1 software (Thermo Fisher Scientific, Waltham, MA, USA) was used for data acquisition and processing, and Origin 8.0 software (Microcal Software Inc., Northampton, MA, USA) was used for plotting.

\section{Results and Discussion}

4.1. Optimization of UHPLC-MS/MS Conditions. The use of formic acid improved the response and peak shape corresponding to FFA but generated ion suppression of CAP, TAP, and FF-which we monitored in the NI mode [28]. Thus, to better separate amphenicols and metabolite residues, we used ACN-0.1 v/v\% formic acid, ACN-0.2 v/v\% formic acid, and ACN-0.3 v/v\% formic acid as the mobile phase for UHPLC-MS/MS analyses in this experiment. The separation effect of the four target compounds was excellent when ACN-water was a mobile phase (Figure 2). The FFA peak became bimodal after adding formic acid to the mobile phase. Therefore, we selected ACN-water as a mobile phase to separate the four analytes.

We directly injected $1 \mu \mathrm{g} / \mathrm{mL}$ of CAP, TAP, FF, FFA, CAP-D5, and FFA-D into the mass spectrometer at a flow rate of $20 \mu \mathrm{L} / \mathrm{min}$, and the instrument scanned in Full Scan Q1 simultaneously in ESI (+) and ESI (-) to determine the precursor ion of each compound. Then the instrument scanned in SIM Q3. For each compound, we optimized two pairs of characteristic ion pairs with the highest response as qualitative and quantitative ion pairs. We also optimized the declustering voltage and collision energy of each ion pair. In doing so, we obtained corresponding mass spectrometry parameters (Table 2).

4.2. Optimization of Sample Preparation. The extraction solvent was ACN, which showed satisfactory recoveries for CAP. However, when the other analytes were introduced in the method, it was necessary to choose the extraction solvent to provide better recoveries rates for all analytes, not just for CAP. The solvent of election was ethyl acetate, which can promote the extraction of all analytes. Moreover, the use of ammonium hydroxide as an additive can promote an 


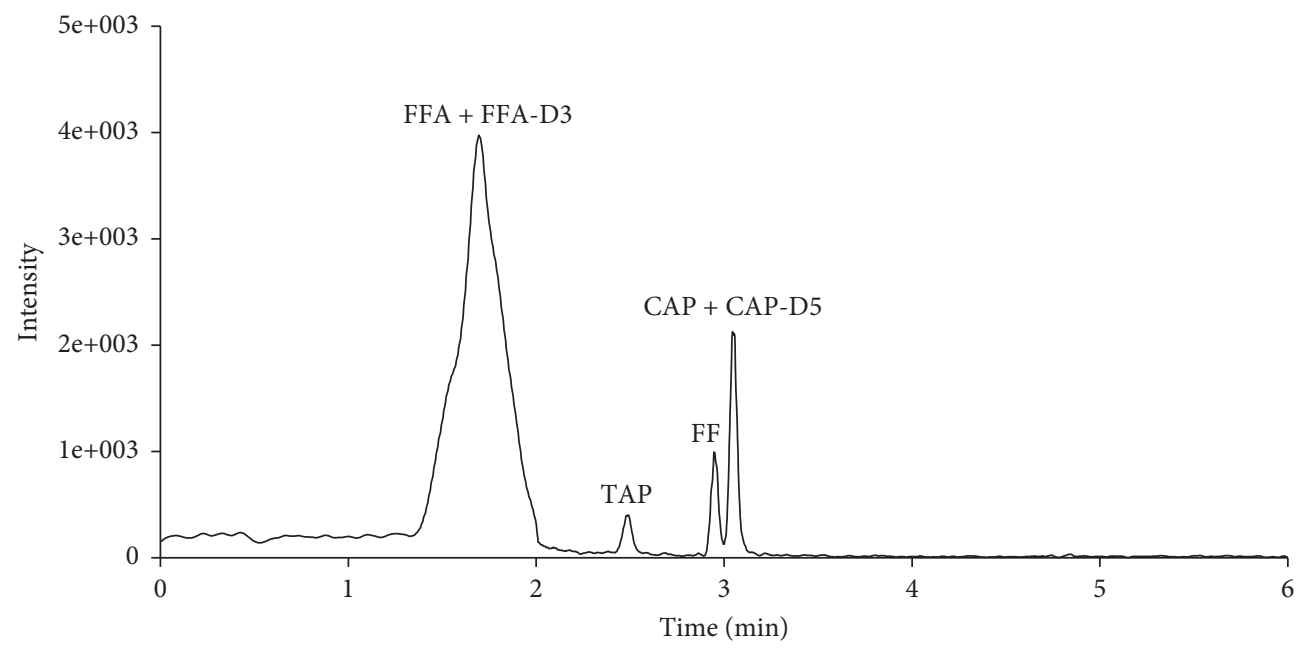

Figure 2: Chromatograms of CAP, TAP, FF, FFA, CAP-D5, and FFA-D3 at $10 \mu \mathrm{g} / \mathrm{L}$ with an ACN-water mobile phase. CAP, chloramphenicol; FF, florfenicol; FFA, florfenicol amine; TAP, thiamphenicol.

TABLE 2: Mass spectrometry conditions and range of relative intensities for each compound.

\begin{tabular}{|c|c|c|c|c|c|}
\hline Analyte & Polarity & Precursor ion $(m / z)$ & Product ion $(\mathrm{m} / \mathrm{z})$ & Declustering voltage $(\mathrm{V})$ & Collision voltage $(\mathrm{V})$ \\
\hline \multirow{2}{*}{ CAP } & \multirow{2}{*}{-} & \multirow{2}{*}{321} & 257 & 113 & -10 \\
\hline & & & $152^{*}$ & 113 & -12 \\
\hline CAP-D5 & - & 326 & 157 & 113 & -16 \\
\hline \multirow{2}{*}{ TAP } & \multirow{2}{*}{-} & \multirow{2}{*}{354} & $290^{*}$ & 290 & -12 \\
\hline & & & 185 & 164 & -10 \\
\hline \multirow{2}{*}{ FF } & \multirow{2}{*}{-} & \multirow{2}{*}{356} & $185^{*}$ & 141 & -20 \\
\hline & & & 119 & 141 & -38 \\
\hline \multirow{2}{*}{ FFA } & \multirow{2}{*}{+} & \multirow{2}{*}{248} & $230^{*}$ & 100 & 10 \\
\hline & & & 130 & 100 & 27 \\
\hline FFA-D3 & + & 251 & 233 & 85 & 10 \\
\hline
\end{tabular}

*Quantification ion. CAP, chloramphenicol; FF, florfenicol; FFA, florfenicol amine; TAP, thiamphenicol.

alkaline environment, which is favorable for the extraction of FFA. Taking into account the extraction efficiency of all the amphenicols and metabolites, it was necessary to choose an extraction solvent that was able to provide better recoveries for all the analytes. Therefore, we chose the following extraction methods for comparison:

(i) Method A: ethyl acetate: ammonium hydroxide (98: 2, v/v), extract twice

(ii) Method B: ACN: ammonium hydroxide (98:2, v/v), extract twice

(iii) Method C: ACN, extract twice

(iv) Method D: ACN: ethyl acetate: ammonium hydroxide (49:49:2, v/v/v), extract twice

(v) Method E: ethyl acetate: ammonium hydroxide (98: 2, v/v), extracted once; and ACN: ammonium hydroxide $(98: 2, \mathrm{v} / \mathrm{v})$, extracted once

After the extraction by method $A$, the recovery of amphenicols and metabolites in the four livestock and poultry meat products was all in the range $60 \%$ to $120 \%$ (Table 3). Therefore, we chose method $\mathrm{A}$ as the optimal extraction method.
Because livestock and poultry meat contain a large quantity of fat and protein and are complex matrices, the samples require further purification after extraction. Therefore, we compared the purification effects of the following columns: HyperSep Retain PEP $(60 \mathrm{mg} / 3 \mathrm{~mL}$; Thermo Fisher Scientific, Waltham, MA, USA), Oasis HLB (200 mg/6 mL; Waters, Milford, MA, USA), and CNW Si SPE $(60 \mathrm{mg} / 3 \mathrm{~mL}$; ANPEL Experimental Technology Co., Shanghai, China). After purification with the CNW Si SPE column, the recovery of amphenicols and metabolites in the four livestock and poultry meat products was in the range from $60 \%$ to $120 \%$ (Table 4 ). Therefore, we chose the CNW Si SPE column as the optimal purification method.

4.3. Linearity of Standard Curves, LOD, and LOQ for Amphenicols and Metabolites. We used CAP-D5 as the internal standard for CAP, TAP; and FF; and FFA-D3 as the internal standard for FFA. In four types of livestock and poultry meat, the method was linear over the range of $0.5-50.0 \mu \mathrm{g} / \mathrm{kg}$ for CAP, TAP, and FF; and of 5.0-200.0 $\mu \mathrm{g} /$ $\mathrm{kg}$ for FFA. The $R^{2}$ was greater than $>0.999$. The LOD and LOQ of the four tested compounds ranged from 
TABLE 3: Recoveries of amphenicols and metabolites under various extraction methods.

\begin{tabular}{|c|c|c|c|c|}
\hline \multirow{2}{*}{ Extraction method } & \multirow{2}{*}{ Matrix } & \multicolumn{3}{|c|}{ Number of amphenicols and metabolites } \\
\hline & & Recovery $<60 \%$ & Recovery $60 \%$ to $120 \%$ & Recovery $>120 \%$ \\
\hline \multirow{4}{*}{ A } & Pork & 0 & 4 & 0 \\
\hline & Beef & 0 & 4 & 0 \\
\hline & Lamb & 0 & 4 & 0 \\
\hline & Chicken & 0 & 4 & 0 \\
\hline \multirow{4}{*}{ B } & Pork & 1 & 1 & 2 \\
\hline & Beef & 1 & 3 & 0 \\
\hline & Lamb & 1 & 1 & 2 \\
\hline & Chicken & 3 & 1 & 0 \\
\hline \multirow{4}{*}{$\mathrm{C}$} & Pork & 1 & 1 & 2 \\
\hline & Beef & 1 & 2 & 1 \\
\hline & Lamb & 1 & 1 & 2 \\
\hline & Chicken & 1 & 3 & 0 \\
\hline \multirow{4}{*}{$\mathrm{D}$} & Pork & 1 & 1 & 2 \\
\hline & Beef & 1 & 2 & 1 \\
\hline & Lamb & 0 & 2 & 2 \\
\hline & Chicken & 0 & 4 & 0 \\
\hline \multirow{4}{*}{$\mathrm{E}$} & Pork & 1 & 2 & 1 \\
\hline & Beef & 1 & 1 & 2 \\
\hline & Lamb & 0 & 2 & 2 \\
\hline & Chicken & 2 & 2 & 0 \\
\hline
\end{tabular}

TABLE 4: Recoveries of amphenicols and metabolites under various purification methods.

\begin{tabular}{|c|c|c|c|c|}
\hline \multirow{2}{*}{ Purification method } & \multirow{2}{*}{ Matrix } & \multicolumn{3}{|c|}{ Number of amphenicols and metabolites } \\
\hline & & Recovery $<60 \%$ & Recovery $60 \%$ to $120 \%$ & Recovery $>120 \%$ \\
\hline \multirow{4}{*}{ HyperSep Retain PEP } & Pork & 1 & 1 & 2 \\
\hline & Beef & 0 & 4 & 0 \\
\hline & Lamb & 1 & 2 & 1 \\
\hline & Chicken & 0 & 2 & 2 \\
\hline \multirow{4}{*}{ Oasis HLB SPE } & Pork & 2 & 0 & 2 \\
\hline & Beef & 0 & 3 & 1 \\
\hline & Lamb & 1 & 2 & 1 \\
\hline & Chicken & 1 & 1 & 2 \\
\hline \multirow{4}{*}{ CNW Si SPE } & Pork & 0 & 4 & 0 \\
\hline & Beef & 0 & 4 & 0 \\
\hline & Lamb & 0 & 4 & 0 \\
\hline & Chicken & 0 & 4 & 0 \\
\hline
\end{tabular}

$0.03-1.50 \mu \mathrm{g} / \mathrm{kg}$ and $0.11-5.0 \mu \mathrm{g} / \mathrm{kg}$ in four types of livestock and poultry meat (Table 5). Compared with other literature reports $[27,28,38]$, this method is fast and has high sensitivity and meets the requirements of the National Food Safety Standard on Maximum Residue Limits for Veterinary Drugs in Foods (GB 31650-2019) in China.

4.4. Recovery and Precision of Amphenicols and Metabolites. To verify the recovery and precision of this method, we spiked blank samples at three concentrations within the ranges $1.0-10.0 \mu \mathrm{g} / \mathrm{kg}$ for CAP, TAP, and FF and $5.0-50.0 \mu \mathrm{g} / \mathrm{kg}$ for FFA ( $n=6$ at each level). Table 6 summarizes the results of the recovery and precision experiments. The average recovery of the four analytes in livestock and poultry meat samples was $71.77 \%$ to $120.30 \%$, with an intraday $\mathrm{RSD}<9.22 \%$ and interday $\mathrm{RSD}<12.30 \%$. Thus, our method has high recovery and good precision and can achieve simultaneous detection of amphenicols and metabolites in livestock and poultry meat.

4.5. Real Sample Analyses. We selected 28 commercial livestock meat, poultry meat, and their products to verify practical applications of our method in real food matrices with possible interferences. We used one positive meat sample as the quality control sample. We detected $1.38 \mu \mathrm{g} / \mathrm{kg}$ of the banned veterinary drug CAP in fresh chicken, $0.35 \mu \mathrm{g} /$ $\mathrm{kg}$ of FF in pork sausage, and $0.54 \mu \mathrm{g} / \mathrm{kg}$ of FF in fried pork jerky (Qingdao, Shandong), yet none of the samples exceeded the MRL of $300 \mu \mathrm{g} / \mathrm{kg}$ for porcine muscle (GB 31650-2019; Table 7). 
TABLE 5: Linearity of standard curve, $R^{2}$, LOD, and LOQ of amphenicols and metabolites in livestock and poultry meat.

\begin{tabular}{|c|c|c|c|c|c|c|}
\hline Matrix & Analyte & Linearity $(\mu \mathrm{g} / \mathrm{kg})$ & Linear equation & $R^{2}$ & LOD $(\mu \mathrm{g} / \mathrm{kg})$ & LOQ $(\mu \mathrm{g} / \mathrm{kg})$ \\
\hline \multirow{4}{*}{ Pork } & CAP & $0.50-50.0$ & $y=0.05933 x+0.02204$ & 0.9996 & 0.11 & 0.36 \\
\hline & TAP & $0.50-50.0$ & $y=0.01864 x+0.02831$ & 0.9992 & 0.12 & 0.37 \\
\hline & $\mathrm{FF}$ & $0.50-50.0$ & $y=0.1219 x+0.05289$ & 0.9998 & 0.03 & 0.11 \\
\hline & FFA & $5.00-200.0$ & $y=0.07707 x-0.00162$ & 0.9991 & 0.60 & 2.00 \\
\hline \multirow{4}{*}{ Beef } & CAP & $0.5-50.0$ & $y=0.06262 x-0.01198$ & 0.9995 & 0.05 & 0.18 \\
\hline & TAP & $0.5-50.0$ & $y=0.01741 x+0.006421$ & 0.9991 & 0.06 & 0.20 \\
\hline & $\mathrm{FF}$ & $0.5-50.0$ & $y=0.1233 x-0.09559$ & 0.9992 & 0.04 & 0.13 \\
\hline & FFA & $5.0-200.0$ & $y=0.06948 x-0.9006$ & 0.9998 & 1.50 & 5.00 \\
\hline \multirow{4}{*}{ Lamb } & CAP & $0.5-50.0$ & $y=0.05427 x+0.07979$ & 0.9995 & 0.06 & 0.21 \\
\hline & TAP & $0.5-50.0$ & $y=0.02154 x+0.02831$ & 0.9992 & 0.06 & 0.21 \\
\hline & $\mathrm{FF}$ & $0.5-50.0$ & $y=0.1553 x+0.2424$ & 0.9998 & 0.04 & 0.12 \\
\hline & FFA & $5.0-200.0$ & $y=0.05435 x-0.1845$ & 0.9992 & 0.50 & 5.00 \\
\hline \multirow{4}{*}{ Chicken } & CAP & $0.5-50.0$ & $y=0.08144 x-0.1681$ & 0.9991 & 0.03 & 0.05 \\
\hline & TAP & $0.5-50.0$ & $y=0.03328 x-0.04784$ & 0.9992 & 0.03 & 0.11 \\
\hline & $\mathrm{FF}$ & $0.5-50.0$ & $y=0.1599 x-0.2144$ & 0.9991 & 0.03 & 0.11 \\
\hline & FFA & $5.0-200.0$ & $y=0.05276 x-0.1651$ & 0.9991 & 1.00 & 3.00 \\
\hline
\end{tabular}

CAP, chloramphenicol; FF, florfenicol; FFA, florfenicol amine; LOD, limit of detection; LOQ, limit of quantification; TAP, thiamphenicol.

TABLE 6: Recovery and precision of amphenicols and metabolites $(n=6)$.

\begin{tabular}{|c|c|c|c|c|c|}
\hline Matrix & Analyte & Added level $(\mu \mathrm{g} / \mathrm{kg})$ & Recovery (\%) & Intraday RSD (\%) & Interday RSD (\%) \\
\hline \multirow{12}{*}{ Pork } & \multirow{3}{*}{ CAP } & 1 & 98.93 & 4.90 & 6.38 \\
\hline & & 5 & 115.81 & 2.16 & 3.71 \\
\hline & & 10 & 113.16 & 5.00 & 5.29 \\
\hline & \multirow{3}{*}{ TAP } & 1 & 95.80 & 8.30 & 9.11 \\
\hline & & 5 & 112.92 & 7.44 & 7.82 \\
\hline & & 10 & 106.31 & 6.93 & 7.92 \\
\hline & \multirow{3}{*}{$\mathrm{FF}$} & 1 & 83.81 & 5.62 & 5.68 \\
\hline & & 5 & 93.02 & 2.82 & 4.59 \\
\hline & & 10 & 95.63 & 1.83 & 4.12 \\
\hline & \multirow{3}{*}{ FFA } & 5 & 97.07 & 2.51 & 6.24 \\
\hline & & 10 & 89.22 & 6.23 & 10.42 \\
\hline & & 50 & 89.61 & 7.93 & 11.94 \\
\hline \multirow{12}{*}{ Beef } & \multirow{3}{*}{ CAP } & 1 & 113.00 & 7.44 & 7.31 \\
\hline & & 5 & 99.73 & 4.41 & 8.24 \\
\hline & & 10 & 93.30 & 2.50 & 10.35 \\
\hline & \multirow{3}{*}{ TAP } & 1 & 87.91 & 5.53 & 9.47 \\
\hline & & 5 & 93.99 & 5.10 & 11.90 \\
\hline & & 10 & 80.10 & 3.02 & 7.10 \\
\hline & \multirow{3}{*}{$\mathrm{FF}$} & 1 & 80.13 & 6.08 & 8.22 \\
\hline & & 5 & 89.50 & 4.90 & 4.93 \\
\hline & & 10 & 96.81 & 2.12 & 2.41 \\
\hline & \multirow{3}{*}{ FFA } & 5 & 86.43 & 9.22 & 9.60 \\
\hline & & 10 & 80.29 & 7.18 & 7.22 \\
\hline & & 50 & 100.22 & 3.90 & 10.8 \\
\hline \multirow{12}{*}{ Lamb } & \multirow{3}{*}{ CAP } & 1 & 97.20 & 1.83 & 2.30 \\
\hline & & 5 & 110.91 & 2.61 & 6.00 \\
\hline & & 10 & 120.30 & 3.88 & 7.43 \\
\hline & \multirow{3}{*}{ TAP } & 1 & 71.77 & 6.15 & 6.20 \\
\hline & & 5 & 105.44 & 4.50 & 4.86 \\
\hline & & 10 & 99.02 & 1.93 & 2.83 \\
\hline & \multirow{3}{*}{$\mathrm{FF}$} & 1 & 81.21 & 6.27 & 12.30 \\
\hline & & 5 & 109.00 & 1.21 & 1.37 \\
\hline & & 10 & 99.03 & 1.90 & 4.19 \\
\hline & \multirow{3}{*}{ FFA } & 5 & 101.14 & 4.58 & 11.00 \\
\hline & & 10 & 103.91 & 5.24 & 8.25 \\
\hline & & 50 & 92.02 & 3.72 & 8.33 \\
\hline
\end{tabular}


TABLE 6: Continued.

\begin{tabular}{|c|c|c|c|c|c|}
\hline Matrix & Analyte & Added level $(\mu \mathrm{g} / \mathrm{kg})$ & Recovery (\%) & Intraday RSD (\%) & Interday RSD (\%) \\
\hline \multirow{12}{*}{ Chicken } & \multirow{3}{*}{ CAP } & 1 & 101.27 & 4.71 & 8.34 \\
\hline & & 5 & 101.05 & 1.63 & 3.60 \\
\hline & & 10 & 97.03 & 5.74 & 5.42 \\
\hline & \multirow{3}{*}{ TAP } & 1 & 84.00 & 6.88 & 11.70 \\
\hline & & 5 & 105.23 & 6.30 & 8.70 \\
\hline & & 10 & 103.54 & 6.91 & 11.02 \\
\hline & \multirow{3}{*}{$\mathrm{FF}$} & 1 & 101.10 & 3.90 & 5.22 \\
\hline & & 5 & 85.44 & 7.16 & 9.70 \\
\hline & & 10 & 82.82 & 4.28 & 4.21 \\
\hline & \multirow{3}{*}{ FFA } & 5 & 108.71 & 6.30 & 5.54 \\
\hline & & 10 & 101.63 & 6.62 & 11.30 \\
\hline & & 50 & 89.90 & 7.30 & 7.64 \\
\hline
\end{tabular}

CAP, chloramphenicol; FF, florfenicol; FFA, florfenicol amine; RSD, relative standard deviation; TAP, thiamphenicol.

TABLE 7: Test results of commercial livestock meat, poultry meat, and their products.

\begin{tabular}{|c|c|c|c|c|c|}
\hline Sample name & Place of origin (China) & CAP & TAP & $\mathrm{FF}$ & FFA \\
\hline Fresh pork (positive control) & Nanjing, Jiangsu & + & + & + & + \\
\hline Fresh pork & Nanjing, Jiangsu & & & & \\
\hline Pork sausage & Linyi, Shandong & & & + & \\
\hline Pork ham & Nanjing, Jiangsu & & & & \\
\hline Fried pork jerky & Zibo, Shandong & & & & \\
\hline Fried pork jerky & Qingdao, Shandong & & & + & \\
\hline Roasted pork jerky & Zhangzhou, Fujian & & & & \\
\hline Roasted pork sausage & Yantai, Shandong & & & & \\
\hline Fresh beef & Nanjing, Jiangsu & & & & \\
\hline Spiced beef & Pingxiang, Jiangxi & & & & \\
\hline Beef sausage & Linyi, Shandong & & & & \\
\hline Fried beef jerky & Bazhong, Sichuan & & & & \\
\hline Fried beef jerky & Zibo, Shandong & & & & \\
\hline Charcoal grilled beef jerky & Tongliao, Nei Mongol & & & & \\
\hline Charcoal grilled beef jerky & Chifeng, Nei Mongol & & & & \\
\hline Fresh lamb & Nanjing, Jiangsu & & & & \\
\hline Boiled lamb & Suzhou, Jiangsu & & & & \\
\hline Spiced lamb & Hulunbuir, Nei Mongol & & & & \\
\hline Fried lamb jerky & Tongliao, Nei Mongol & & & & \\
\hline Fried lamb jerky & Bayannaoer, Nei Mongol & & & & \\
\hline Roast leg of lamb & Hulunbuir, Nei Mongol & & & & \\
\hline Roast leg of lamb & Xilin Gol League, Nei Mongol & & & & \\
\hline Fresh chicken & Nanjing, Jiangsu & + & & & \\
\hline Instant chicken breast & Pingxiang, Jiangxi & & & & \\
\hline Chicken sausage & Linyi, Shandong & & & & \\
\hline Crispy chicken & Nanjing, Jiangsu & & & & \\
\hline Fried chicken & Nanjing, Jiangsu & & & & \\
\hline Roast chicken & Yantai, Shandong & & & & \\
\hline Beggar's chicken & Hangzhou, Zhejiang & & & & \\
\hline
\end{tabular}

+, detected; no entry indicates not detected. CAP, chloramphenicol; FF, florfenicol; FFA, florfenicol amine; TAP, thiamphenicol.

\section{Conclusions}

Amphenicols are widely used in livestock and poultry breeding and production, but illegal or noncompliant use may occur. This causes amphenicol residues to accumulate in the body of livestock and poultry animals. Such accumulation is a threat to livestock and poultry food safety, as well as human health. Therefore, we developed a detection method for amphenicols and metabolites in livestock meat, poultry meat, and their products. By optimizing MS, LC, and sample pretreatment conditions, we established a UHPLCMS/MS detection method that can simultaneously detect amphenicols and metabolites in livestock meat, poultry meat, and their products. The peak time of the analytes was within $5 \mathrm{~min}$; the LOD was $0.03-1.50 \mu \mathrm{g} / \mathrm{kg}$; the LOQ was $0.05-5.00 \mu \mathrm{g} / \mathrm{kg}$; the recovery of spiked samples at low, medium, and high concentrations was between $71.77 \%$ and $120.30 \%$; the intraday RSD was $<9.22 \%$, and the interday RSD was $<12.30 \%$. Thus, this method has a short analysis time, a high sensitivity, and a good repeatability. We have 
thus provided scientific support and technical assurance for reliable detection of amphenicols and metabolites in animalderived foods for food safety monitoring.

\section{Data Availability}

The original data used to support the findings of this study can be obtained from the corresponding author upon request.

\section{Conflicts of Interest}

The authors declare that there are no conflicts of interest regarding the publication of this study.

\section{Acknowledgments}

The authors thank Qingqin Lü for her valuable advice on UHPLC-MS/MS analysis. This research was financially supported by grants from the National Key R\&D Program of China (2018YFC1602804) and a special intergovernmental project for International Cooperation in Science, Technology and Innovation (2019YFE0103800).

\section{References}

[1] G. Zhou, W. Zhang, and X. Xu, "China's meat industry revolution: challenges and opportunities for the future," Meat Science, vol. 92, no. 3, pp. 188-196, 2012.

[2] M. Pastor-Belda, N. Campillo, N. Arroyo-Manzanares, M. Hernández-Córdoba, and P. Viñas, "Determination of amphenicol antibiotics and their glucuronide metabolites in urine samples using liquid chromatography with quadrupole time-of-flight mass spectrometry," Journal of Chromatography B, vol. 1146, Article ID 122122, 2020.

[3] N. R. Barveen, T.-J. Wang, and Y.-H. Chang, "Photochemical decoration of silver nanoparticles on silver vanadate nanorods as an efficient SERS probe for ultrasensitive detection of chloramphenicol residue in real samples," Chemosphere, vol. 275, p. 130115, 2021.

[4] L. R. Guidi, F. A. Santos, A. C. S. R. Ribeiro, C. Fernandes, L. H. M. Silva, and M. B. A. Gloria, "A simple, fast and sensitive screening LC-ESI-MS/MS method for antibiotics in fish," Talanta, vol. 163, pp. 85-93, 2017.

[5] J. P. Cerutti, V. Aiassa, M. A. Fernández, M. R. Longhi, M. A. Quevedo, and A. Zoppi, "Structural, physicochemical and biological characterization of chloramphenicol multicomponent complexes," Journal of Molecular Liquids, vol. 331, p. 115761, 2021.

[6] P. Ma, H. Guo, N. Duan et al., "Label free structure-switching fluorescence polarization detection of chloramphenicol with truncated aptamer," Talanta, vol. 230, p. 122349, 2021.

[7] L. R. Guidi, P. A. S. Tette, M. B. A. Gloria, and C. Fernandes, "A simple and rapid LC-MS/MS method for the determination of amphenicols in Nile tilapia," Food Chemistry, vol. 262, pp. 235-241, 2018.

[8] F. Wang, W. Wang, S. Yuan, W. Wang, and Z.-H. Hu, "Comparison of UV/H2O2 and UV/PS processes for the degradation of thiamphenicol in aqueous solution," Journal of Photochemistry and Photobiology A: Chemistry, vol. 348, pp. 79-88, 2017.

[9] R. Li, Z.-J. Lin, J.-Y. Yang et al., “An indirect competitive enzyme-linked immunosorbent assay for simultaneous determination of florfenicol and thiamphenicol in animal meat and urine," Chinese Journal of Analytical Chemistry, vol. 46, no. 8, pp. 1321-1328, 2018.

[10] G. Wang, B. Wang, X. Zhao et al., "Determination of thiamphenicol, florfenicol and florfenicol amine residues in poultry meat and pork via ASE-UPLC-FLD," Journal of Food Composition and Analysis, vol. 81, pp. 19-27, 2019.

[11] A. Anadón, M. A. Martínez, M. Martínez et al., "Plasma and tissue depletion of florfenicol and florfenicol-amine in chickens," Journal of Agricultural and Food Chemistry, vol. 56, no. 22, pp. 11049-11056, 2008.

[12] L. Campone, R. Celano, A. L. Piccinelli et al., "Ultrasound assisted dispersive liquid-liquid microextraction for fast and accurate analysis of chloramphenicol in honey," Food Research International, vol. 115, pp. 572-579, 2019.

[13] L. R. Guidi, P. A. S. Tette, C. Fernandes, L. H. M. Silva, and M. B. A. Gloria, "Advances on the chromatographic determination of amphenicols in food," Talanta, vol. 162, pp. 324-338, 2017.

[14] X. Ma, H. Li, S. Qiao et al., "A simple and rapid sensing strategy based on structure-switching signaling aptamers for the sensitive detection of chloramphenicol," Food Chemistry, vol. 302, p. 125359, 2020.

[15] J. Ding, Q. Li, X. Xu et al., “A wheat straw cellulose-based hydrogel for $\mathrm{Cu}$ (II) removal and preparation copper nanocomposite for reductive degradation of chloramphenicol," Carbohydrate Polymers, vol. 190, pp. 12-22, 2018.

[16] Ministry of Agriculture and Rural Affairs of the People's Republic of China, National Health Commission of the People's Republic of China, State Administration for Market Regulation, and National Standard of the People's Republic of China, National Food Safety Standard on Maximum Residue Limits for Veterinary Drugs in Foods, GB31650-2019, ChemLinked, Beijing, China, 2019, https://www.aqsc.agri.cn/ tzgg/201910/P020191012570175865095.pdf.

[17] Commission European, Commission Regulation (EU) No 37/ 2010 of 22 December 2009 on Pharmacologically Active Substances and their Classification Regarding Maximum Residue Limits in Foodstuffs of Animal Origin (Text with EEA Relevance), Official Journal of the European Union, Luxembourg, Europe, 2009, https:/ec.europa.eu/health/sites/default/files/ files/eudralex/vol-5/reg_2010_37/reg_2010_37_en.pdf.

[18] A. S. Sadeghi, N. Ansari, M. Ramezani et al., "Optical and electrochemical aptasensors for the detection of amphenicols," Biosensors and Bioelectronics, vol. 118, pp. 137-152, 2018.

[19] H. Li, W. Geng, M. M. Hassan et al., "Rapid detection of chloramphenicol in food using SERS flexible sensor coupled artificial intelligent tools," Food Control, vol. 128, p. 108186, 2021.

[20] L. V. De Faria, T. P. Lisboa, N. D. S. Campos et al., "Electrochemical methods for the determination of antibiotic residues in milk: a critical review," Analytica Chimica Acta, vol. 1173, p. 338569, 2021.

[21] A. S. Sadeghi, M. Mohsenzadeh, K. Abnous, S. M. Taghdisi, and M. Ramezani, "Development and characterization of DNA aptamers against florfenicol: fabrication of a sensitive fluorescent aptasensor for specific detection of florfenicol in milk," Talanta, vol. 182, pp. 193-201, 2018.

[22] N. Sai, Y. Chen, N. Liu et al., "A sensitive immunoassay based on direct hapten coated format and biotin-streptavidin system for the detection of chloramphenicol," Talanta, vol. 82, no. 4, pp. 1113-1121, 2010. 
[23] A. Yibar, F. Cetinkaya, and G. E. Soyutemiz, "ELISA screening and liquid chromatography-tandem mass spectrometry confirmation of chloramphenicol residues in chicken muscle, and the validation of a confirmatory method by liquid chromatography-tandem mass spectrometry," Poultry Science, vol. 90, no. 11, pp. 2619-2626, 2011.

[24] A. Posyniak, J. Zmudzki, and J. Niedzielska, "Evaluation of sample preparation for control of chloramphenicol residues in porcine tissues by enzyme-linked immunosorbent assay and liquid chromatography," Analytica Chimica Acta, vol. 483, no. $1-2$, pp. $307-311,2003$.

[25] V. Samanidou, L.-D. Galanopoulos, A. Kabir, and K. G. Furton, "Fast extraction of amphenicols residues from raw milk using novel fabric phase sorptive extraction followed by high-performance liquid chromatography-diode array detection," Analytica Chimica Acta, vol. 855, pp. 41-50, 2015.

[26] F. Moragues, C. Igualada, and N. León, "Validation of the determination of chloramphenicol residues in animal feed by liquid chromatography with an ion trap detector based on European decision 2002/657/EC," Food Analytical Methods, vol. 5, no. 3, pp. 416-421, 2011.

[27] F. Moragues, C. Igualada, and N. León, "Confirmatory method for the determination of amphenicols in muscle and kidney of several animal species," Food Analytical Methods, vol. 10, no. 3, pp. 610-617, 2016.

[28] H.-Y. Liu, S.-L. Lin, and M.-R. Fuh, "Determination of chloramphenicol, thiamphenicol and florfenicol in milk and honey using modified QuEChERS extraction coupled with polymeric monolith-based capillary liquid chromatography tandem mass spectrometry," Talanta, vol. 150, pp. 233-239, 2016.

[29] R. W. Fedeniuk, D. McKenzie, M. Mizuno, C. Neiser, C. O’Byrne, and B. Shurmer, "Development and validation of determinative and confirmatory LC-MS/MS methodologies for total florfenicol and tulathromycin residues in bovine, equine and porcine kidney, liver and muscle tissues," Journal of Chromatography B, vol. 983-984, pp. 1-9, 2015.

[30] X.-D. Pan, P.-G. Wu, W. Jiang, and B.-J. Ma, "Determination of chloramphenicol, thiamphenicol, and florfenicol in fish muscle by matrix solid-phase dispersion extraction (MSPD) and ultra-high pressure liquid chromatography tandem mass spectrometry," Food Control, vol. 52, pp. 34-38, 2015.

[31] S. Zhang, F. Sun, J. Li, L. Cheng, and J. Shen, "Simultaneous determination of florfenicol and florfenicol amine in fish, shrimp, and swine muscle by gas chromatography with a microcell electron capture detector," Journal of AOAC International, vol. 89, no. 5, pp. 1437-1442, 2006.

[32] Y. Yikilmaz and A. Filazi, "Detection of florfenicol residues in salmon trout via GC-MS," Food Analytical Methods, vol. 8, no. 4, pp. 1027-1033, 2014.

[33] W.-L. Liu, R.-J. Lee, and M.-R. Lee, "Supercritical fluid extraction in situ derivatization for simultaneous determination of chloramphenicol, florfenicol and thiamphenicol in shrimp," Food Chemistry, vol. 121, no. 3, pp. 797-802, 2010.

[34] H. Kikuchi, T. Sakai, R. Teshima, S. Nemoto, and H. Akiyama, "Total determination of chloramphenicol residues in foods by liquid chromatography-tandem mass spectrometry," Food Chemistry, vol. 230, pp. 589-593, 2017.

[35] S. Saito-Shida, K. Shiono, J. Narushima, S. Nemoto, and H. Akiyama, "Determination of total florfenicol residues as florfenicol amine in bovine tissues and eel by liquid chromatography-tandem mass spectrometry using external calibration," Journal of Chromatography B, vol. 1109, pp. 37-44, 2019.
[36] X. Xie, B. Wang, M. Pang et al., "Quantitative analysis of chloramphenicol, thiamphenicol, florfenicol and florfenicol amine in eggs via liquid chromatography-electrospray ionization tandem mass spectrometry," Food Chemistry, vol. 269, pp. 542-548, 2018.

[37] Z. Xiao, R. Song, Z. Rao et al., "Development of a subcritical water extraction approach for trace analysis of chloramphenicol, thiamphenicol, florfenicol, and florfenicol amine in poultry tissues," Journal of Chromatography A, vol. 1418, pp. 29-35, 2015.

[38] U.S. Department of Health and Human Services, U.S. Food and Drug Administration, Center for Drug Evaluation and Research (CDER), and Center for Veterinary Medicine (CVM), Bioanalytical Method Validation: Guidance for Industry (2018), U.S. Food and Drug Administration, Washington, DC, USA, 2001, https://www.fda.gov/media/70858/ download. 